\title{
Configuración de la acreditación de la calidad como campo de estudio. Una revisión sistemática de la investigación internacional (1998-2016)
}

\author{
Sandra Guzmán-Puentes y René Guevara-Ramírez
}

\section{RESUMEN}

Este artículo realiza una revisión sistemática de las investigaciones publicadas en revistas indexadas sobre acreditación de la calidad de la educación superior para el periodo 1998-2016. ${ }^{1}$ Establece el posicionamiento de la acreditación en la agenda investigativa por país, región e institución; también identifica ocho comunidades académicas conformadas a partir de redes de colaboración. Desde la teoría de Bourdieu, analiza el reconocimiento de los agentes universitarios acerca de la acreditación como un juego digno de ser jugado para mantenerse y mejorar la posición al interior del campo de la educación superior. Se argumenta que las condiciones objetivas que circunscriben estos procesos se relacionan con indicadores de planes de gobierno y demandas de agencias internacionales de financiamiento, lo que pone en riesgo la práctica académica. Se concluye la innecesaria acción de la acreditación para el logro de la calidad educativa, y se plantean desafíos de transformación para este tipo de políticas.

Palabras clave: educación superior, acreditación, calidad de la educación, evaluación, políticas públicas.

Sandra Guzmán-Puentes Colombiana. Candidata a Doctora en Educación, Universidad Pedagógica Nacional, Colombia. Profesional, Dirección de Asuntos Profesorales, Vicerrectoría Académica, Pontificia Universidad Javeriana, Colombia. Temas de investigación: evaluación de la educación superior, políticas educativas, desarrollo institucional y deserción estudiantil. ORCID: https://orcid.org/0000-0002$8492-6905$

\section{René Guevara-Ramírez} Posgrados, Universidad Pedagógica Nacional, Bogotá, Colombia. Temas de investigación: estudios sobre cambio institucional en universidades públicas, gobernanza universitaria, políticas de educación superior. ORCID: https://orcid.org/0000-0002-1635-4407

\footnotetext{
${ }^{1}$ Agradecimiento a los revisores anónimos por sus fructíferos comentarios que contribuyeron a mejorar la versión final del artículo. El artículo hace parte de la investigación doctoral titulada "La configuración de las prácticas de los profesores universitarios y su relación con las políticas institucionales de aseguramiento de la calidad de la educación superior" del programa de Doctorado Interinstitucional de Educación(DIE) de la Universidad Pedagógica Nacional, Bogotá, Colombia.
} 


\title{
Configuração da aprovação da qualidade como campo de estudo. Uma revisão sistemática da pesquisa internacional (1998-2016)
}

\section{RESUMO}

Este artigo realiza uma revisão sistemática das pesquisas publicadas em revistas indexadas sobre aprovação da qualidade da educação superior para o período 1998-2016. Estabelece o posicionamento da aprovação na agenda investigativa por país, região e instituição; também identifica oito comunidades acadêmicas conformadas a partir de redes de colaboração. Desde a teoria de Bourdieu, analisa o reconhecimento dos agentes universitários sobre a aprovação como um jogo digno de ser jogado para manter-se e melhorar a posição no interior do campo da educação superior. Se argumenta que as condições objetivas que circunscrevem estes processos se relacionam com indicadores de planos de governo e demandas de agências internacionais de financiamento, o que põe em risco a prática académica. Se conclui a inecessária ação da aprovação para a conquista da qualidade educativa, e se apresenta desafios de transformação para este tipo de políticas.

Palavras chave: educação superior, aprovação, qualidade da educação, avaliação, políticas públicas.

\section{Shaping quality accreditation as a field of study. A systematic review of international research} (1998-2016)

\begin{abstract}
This article conducts a systematic review of research published in indexed journals on higher education quality accreditation for the period 1998-2016. It identifies the positioning of accreditation in the research agenda by country, region and institution; it also recognizes eight academic communities formed from collaborative networks. From Bourdieu's theory, it analyzes the recognition of university agents about accreditation as a game worth playing to maintain and improve the position within the field of higher education. The authors allege that the objective conditions that circumscribe these processes are related to indicators of government plans and demands of international funding agencies, which puts academic practice at risk. They conclude that accreditation is unnecessary for the achievement of educational quality and propose challenges for the transformation of this type of policies.
\end{abstract}

Key words: higher education, accreditation, quality of education, assessment, public policies. 


\section{Introducción}

En distintos países, se identificó un cúmulo de estudios que ubican el origen de la acreditación de la calidad en la década de los setenta, bajo la necesidad de contar con un mecanismo de control y regulación ante la proliferación de nueva oferta académica de disímil origen y naturaleza, ocasionada por demandas relacionadas con el incremento de los índices de cobertura educativa para atender necesidades de desarrollo económico. Al respecto Brock (2006) menciona que estos conceptos de acreditación y de proliferación de la oferta no son conceptos recientes, en realidad, han sido fenómenos inherentes al surgimiento del mismo concepto de universidad en el siglo XI, con la concesión desmedida de licencias para la conformación de los denominados studium generale, corporaciones de maestros y discípulos, que crearon escuelas universitarias de ciencia y tecnología, algunas de las cuales más tarde se denominaron universidades, sobre las cuales se expondría la necesidad de regular su oferta y funcionamiento por lo que fue necesario otorgar una distinción exclusiva por parte de un monarca para enseñar a un nivel especifico, lo que entenderíamos hoy como el proceso de acreditación de programas. De igual manera, el autor menciona que a través de la historia el quehacer universitario ha sido esencialmente instrumental y ha padecido distintas formas de control y supervisión por parte de diversos estamentos religiosos, políticos o económicos, quienes siempre han ejercido un control no sólo sobre la oferta universitaria, sino sobre el contenido y práctica de la enseñanza, y el reconocimiento de los títulos, para cumplir con requisitos referidos especialmente al suministro de mano de obra educada y académicamente capacitada que les permitiera conservar sus intereses en sociedades cada vez más sofisticadas y cambiantes.

Así, lo que vemos en la actualidad no es otra cosa que la versión actualizada de lo que ha sido una tradición en desregulación del mercado, debido al surgimiento desmesurado de instituciones privadas y estatales que, luego de someterse al proceso de licenciamiento para obtener su autonomía institucional, abrieron numerosas sedes y programas de dudosa calidad, razón que daría lugar a la implementación de los denominados sistemas de aseguramiento de la calidad. Acorde con Geoffroy (2013), actualmente este control de la oferta educativa, no es otra cosa que políticas reactivas a las demandas de agencias externas relacionadas con ayudas públicas a la educación superior para aquellos países e instituciones que apoyen el desarrollo y crecimiento económico a nivel regional. Dichas ayudas se fundamentan en el incremento de los índices de cobertura, especialmente para contar con una mayor proporción de mano de obra calificada. Ejemplo de ello, la propuesta de la Organización para la Cooperación y el Desarrollo Económicos (OCDE) de elevar las tasas de matrícula por encima del $40 \%$, la cual fue respaldada por la UNESCO en 2003, bajo el planteamiento de que el acceso a la educación sería el elemento diferenciador entre países desarrollados y los menos desarrollados (Uvalić-Trumbić, 2006), lo que ubicaría a la educación superior como un escenario para la generación de nuevas ideas y de formación de personas que impacten el desarrollo del contexto local y regional (Rodman et al., 2013).

Así mismo, los procesos de acreditación también son considerados un instrumento que permite a la educación superior hacer frente a las transformaciones que tienen lugar en la economía, la sociedad y la civilización, esto, evidenciado en demandas como las del Banco Interamericano de Desarrollo (BID) con su exigencia para instaurar sistemas de aseguramiento de la calidad comparables como condición para financiar grandes proyectos nacionales (UvalićTrumbić, 2006), y serían condición para el establecimiento de tratados internacionales que favorecerían la circulación internacional de profesionales (Cabalin, 2015). No obstante, Ginkel y Dias (2007) sustentarían que la implementación de sistemas de acreditación ha desencadenado una creciente apreciación de la 
educación superior como mercado, poniendo en riesgo los valores fundamentales de la educación superior, tales como el concepto de la educación superior como un bien público y una responsabilidad pública, y la educación superior basada en el mérito como uno de los derechos humanos básicos.

\section{La teoría de la acción social, un marco conceptual para el análisis de la acreditación}

Como problemática teórica, el análisis reconoce las condiciones objetivas que han dado lugar a la implementación de políticas de acreditación al interior de las instituciones de educación superior (IES), tratando de comprender cómo reaccionan las instituciones a dichas políticas y por qué se esfuerzan por conseguir la acreditación. Para ello, reconoce en la acción de los agentes dos formas de ver el mundo, el sentido objetivo que refiere a las estructuras sociales externas e independientes de la conciencia y de la voluntad de los agentes, es decir a la estructura del espacio universitario y sus políticas, y el sentido vivido orientado al de los agentes, cómo se representan, sienten, piensan, creen e incorporan este tipo de procesos y eventualmente lo convierten en una práctica. Por ello, la investigación toma como soporte para el análisis, estos dos "modos de conocimiento teórico" que propone Pierre Bourdieu con su teoría de la acción social, a través de la cual nos invita a comprender la realidad, mediante una relación construida entre lo social, representado en condiciones objetivas y de las estructuras sociales internalizadas, y lo social hecho cuerpo, es decir, las estructuras incorporadas en los agentes (Gutiérrez, 2012). Así, a través de la exploración de los resultados de investigaciones realizadas, se intenta identificar tanto el proceder de las instituciones relacionadas con la forma en que reconocen las posibilidades, las limitaciones e implicancias de la política de acreditación, como los esquemas de percepción, de apreciación y de acción interiorizados por los agentes universitarios.
Para ello, se reconoce que "la acción se relaciona con la externalización subjetiva del agente, así como con la inscripción de la acción en un sistema de relaciones sociales" (Giddens, 1984: 42), por lo que si se quiere indagar por la acción de los agentes en el marco de los procesos de acreditación, sería necesario tomar en cuenta la noción de acción social definida por Weber como "aquella conducta en la que el significado que a ella atribuye el agente o agentes entraña una relación con respecto a la conducta de otra u otras personas y en las que tal relación determina el modo en que procede dicha relación" (Giner, 2001: 283). En consecuencia, en el caso de la calidad educativa, el análisis considera aquellas investigaciones que hablan de la acción de los agentes desde el punto de vista de la racionalización y la reflexión que hacen sobre las falencias en las prácticas universitarias y la forma en que los procesos de acreditación han introducido cambios para mejorarlas.

Así mismo, el análisis incorpora la asunción de espacio social de Bourdieu, en la medida en que podemos describir el campo de la acreditación como un campo de fuerzas, es decir, "como un conjunto de relaciones de fuerzas objetivas que se imponen a todos los que entran en ese campo y que son irreductibles a las intenciones de los agentes individuales o incluso a las interacciones directas entre los agentes" (Bourdieu, 1989: 27). Dicho espacio se define, entre otras cosas, por la existencia de directivos universitarios y demás agentes universitarios, que poseen el conocimiento y el reconocimiento del valor de la acreditación y de las reglas para el logro de este tipo de reconocimientos, es decir, como lo menciona Bourdieu (1990), en la cual tendría que haber agentes que están dispuestos a jugar el juego y reconocer leyes inmanentes del mismo.

Se entiende que los agentes universitarios manifiestan la existencia de un sentido del juego, en la medida que luchan no sólo por mantener su posición al interior del sistema universitario, sino además por lograr cada vez mayor reconocimiento y prestigio 
sobre la calidad, por lo que la acreditación vendría a constituir el capital simbólico por el cual todos quieren luchar.

En consecuencia, la universidad pensada como un espacio social, implica pensarla en términos relacionales, dice Bourdieu, al pensarla como una red o una configuración de relaciones objetivas entre posiciones que están objetivamente definidas, en su existencia y por su situación presente y potencial al interior del espacio social y cuya distribución de capital ordena el acceso a ventajas específicas que están en juego en el campo, así como por su relación objetiva con otras posiciones (Bourdieu y Wacquant, 2005). Así, estos espacios poseen unas propiedades que pueden ser analizadas independientemente de las características de sus ocupantes (Bourdieu, 1988: 113-114), por lo que sumada cuenta, se trata de conocer y reconocer a través de estas investigaciones las estructuras que determinan los principios de acción en las prácticas universitarias en el logro de la acreditación.

\section{Método}

Se revisó sistemáticamente la literatura sobre publicaciones científicas durante el periodo 1998- 2016. ${ }^{2}$ La búsqueda se centró en artículos de revistas indexadas en las bases bibliográficas Scopus, Web of Science y SciELO con los términos: "higher education", "quality", "assurance", "accreditation" y "policies". Se consideraron como criterios de exclusión las publicaciones clasificadas por las revistas como: reportes breves, documentos de reflexión, cartas al editor y ponencias, para lo cual se realizó un filtro por título y descriptores. Este proceso permitió identificar, en primera instancia, que aunque las bases de datos recuperan un alto número de publicaciones relacionadas con el tema, en realidad las investigaciones que abordan problemáticas en torno a la acreditación de la calidad son limitadas.
El resultado de la búsqueda general arrojó 5263 artículos iniciales, a partir de los cuales se procedió a realizar una delimitación de búsqueda por temáticas y descriptores dando como resultado 340 artículos para analizar. A partir de estos últimos se elaboró un análisis de redes de colaboración entre países, instituciones y autores mediante la identificación del número de artículos publicados por país, las instituciones participantes, las redes de publicación entre instituciones, los autores y las temáticas comunes. Para este propósito, se emplearon las herramientas de análisis y visualización de información: VantagePoint, Gephi y Tableau. En posterior lectura detallada de los resúmenes se delimitó una muestra de 58 artículos que estaban directamente relacionados con las categorías de análisis propuestas, con los cuales se realizó un análisis de contenido a partir de las cuatro categorías propuestas: a) la lucha de las instituciones por conseguir la acreditación; b) los capitales que determinan su obtención; c) su interiorización en las prácticas docentes; y d) las críticas y los desafíos de transformación.

\section{Resultados \\ Configuración de la acreditación de la calidad como campo de investigación}

En este apartado, se realizó una lectura de indicadores de actividad que permite visualizar el estado de investigación sobre el tema categorizando la información de la siguiente manera: el número y distribución de publicaciones por instituciones; la productividad o número de trabajos por autor, revista o institución, la dispersión de las publicaciones y el análisis de las contribuciones sobre un tema, además de los núcleos de autores y los indicadores de cooperación. Con ello se intenta describir cómo se ha desarrollado la actividad investigativa, a nivel de países, instituciones y temas, en torno a la

${ }_{2}^{2} 1998$ es el año en el cual inicia el proceso de acreditación en Colombia. El año 2017 no fue incluido en el análisis debido a que la información estaba siendo normalizada por los sistemas de indexación mencionados durante el desarrollo de la presente investigación. 
acreditación de la calidad de la educación superior, a fin de establecer las redes de colaboración existentes, las comunidades académicas emergentes, y las temáticas comunes de trabajo.

Se logró establecer que, a pesar de que el tema de la acreditación aún no constituye un avance desde el análisis disciplinar, si se ha configurado como campo de estudio, en tanto se han conformado comunidades académicas que han establecido redes entre investigadores, universidades y países trabajando temas de interés común para el mejoramiento de la calidad educativa y el desarrollo de los países.

A continuación, se presenta la dinámica de publicación en torno al tema de acreditación de la calidad y se indica la participación de artículos por país, universidades o IES que han desarrollado investigaciones temáticas. Se destaca que, aunque es incipiente el número de publicaciones que hablan de manera particular sobre la forma como se han interiorizado las políticas de acreditación en los sistemas universitarios, las instituciones y los agentes, es incipiente en relación con otros temas referidos a la calidad educativa, sus mayores contribuciones corresponden principalmente a América, Europa y Asia, como se puede observar en las figuras 1 y 2 .

El área geográfica que presenta un marcado liderazgo es América, con 172 publicaciones, teniendo el mayor aporte a nivel de país, Estados Unidos, con 67 publicaciones a través de la participación de 38 universidades, especialmente con los aportes de la Universidad de Harvard, la Universidad de California y la Universidad de Washington. En Latinoamérica se encontraron 60 universidades con 98 artículos sobre acreditación de la calidad de la educación superior. Se destaca Chile con 33 publicaciones en 12 IES de las 53 existentes, sobresaliendo de manera especial las universidades de Tarapacá de Arica, Chile y Pontificia Universidad Católica de Chile que aportan el análisis más exhaustivo en relación con los efectos en el país y la región. Colombia, con 15 artículos publicados en 10 IES de las 289 existentes; se destacan la Universidad Nacional de Colombia, la Universidad de Antioquia y la Universidad del Norte. Sorprende Cuba con 13 publicaciones y cuatro universidades de 21 existentes; México con 10 artículos en siete de 430 IES y Brasil con 9 publicaciones en 11 de 366 universidades. Se notó un escaso desarrollo de la investigación en el tema en países como Ecuador, Venezuela, Bolivia y Perú, entre otros.

En Europa, sobresalen España y el Reino Unido con 17 y 13 publicaciones respectivamente. En España, están la Universidad Politécnica de Madrid y la Universidad Autónoma de Barcelona. Por su parte, en Reino Unido se destacan la Universidad de Oxford, Imperial College London y la Universidad de Birmingham. Portugal presenta ocho publicaciones provenientes de seis universidades. Llama la atención encontrar institutos o agencias dedicadas al estudio del aseguramiento de acreditación de la educación superior como The Learning laboratories Copenhaguen (Dinamarca), The Ontario Institute for Studies in Education (Canadá), La Agencia de Aseguramiento de Acreditación de la Educación Superior (España), el Centro de Investigação de Políticas do Ensino Superior (Portugal) y la UNESCO (Francia).

Con respecto al resto del mundo, se evidenció una participación escasa en países de Medio Oriente. No obstante, el este asiático, comprendido por China, Japón y Corea del Sur mostró algunos avances de las universidades: Open University of China, Beijing Normal University, Pekin University, University of Singapore, Fujen Catholic University y National Taipei University of Education. Se halló un interés particular por la temática en Australia con cinco publicaciones de las IES: The University of Sydney, Monash University, University of New South Wales (UNSW) Australia y University of Melbourne. 


\section{Figura 1. Número de artículos e instituciones interesadas en investigar el tema de acreditación de la calidad en la educación superior: Latinoamérica}

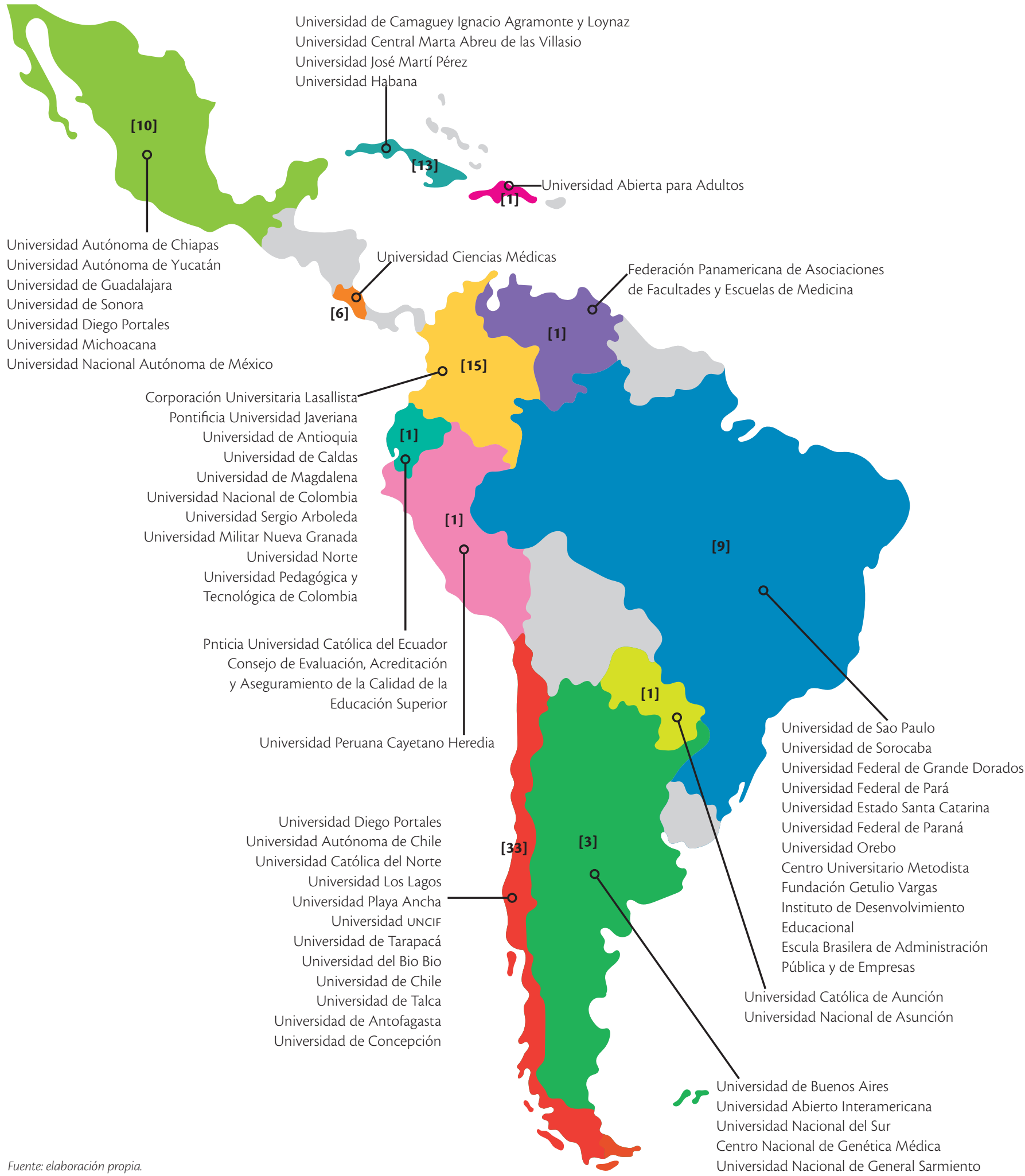


Figura 2. Número de artículos e instituciones interesadas en investigar el tema de acreditación de la calidad en la educación superior: Estados Unidos y Europa

Toronto University

Simon Fraser University

Ontario Institute for Studies in Education

McGill University

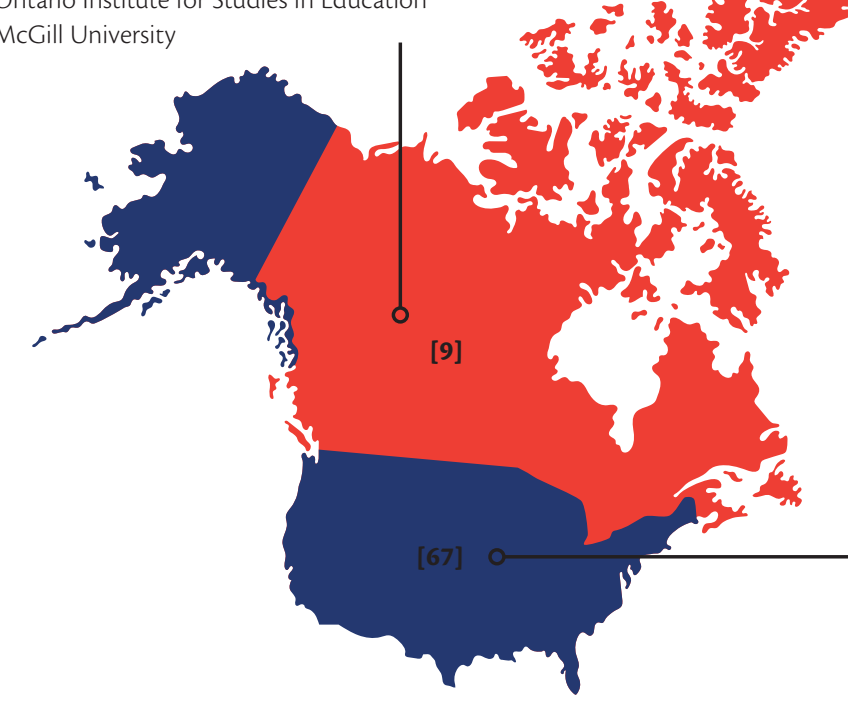

ormas Jefferson University Mssachusetts University Michigan University Johns Hopkins University Maryland University

Minessota University

North Carolina University

Penn University

Wisconsin University

Acadia University

Boise State University

Duke University

Emory University

George Mason University

Harvard University

Indiana University

Western Michigan University

Yale University

lowa State University

Louisiana State University

Northwestern University

Oregon Health and Science

University

Purdue University

Tulane University

University of Alabama

University of Arizona

University of California

San Francisco

University of Colorado Denver

University of Connecticut

University of Florida

University of Hawaii

University of Kansas

University of Oklahoma

University of Texas

Virginia Commonwealth University

Western Illinois University

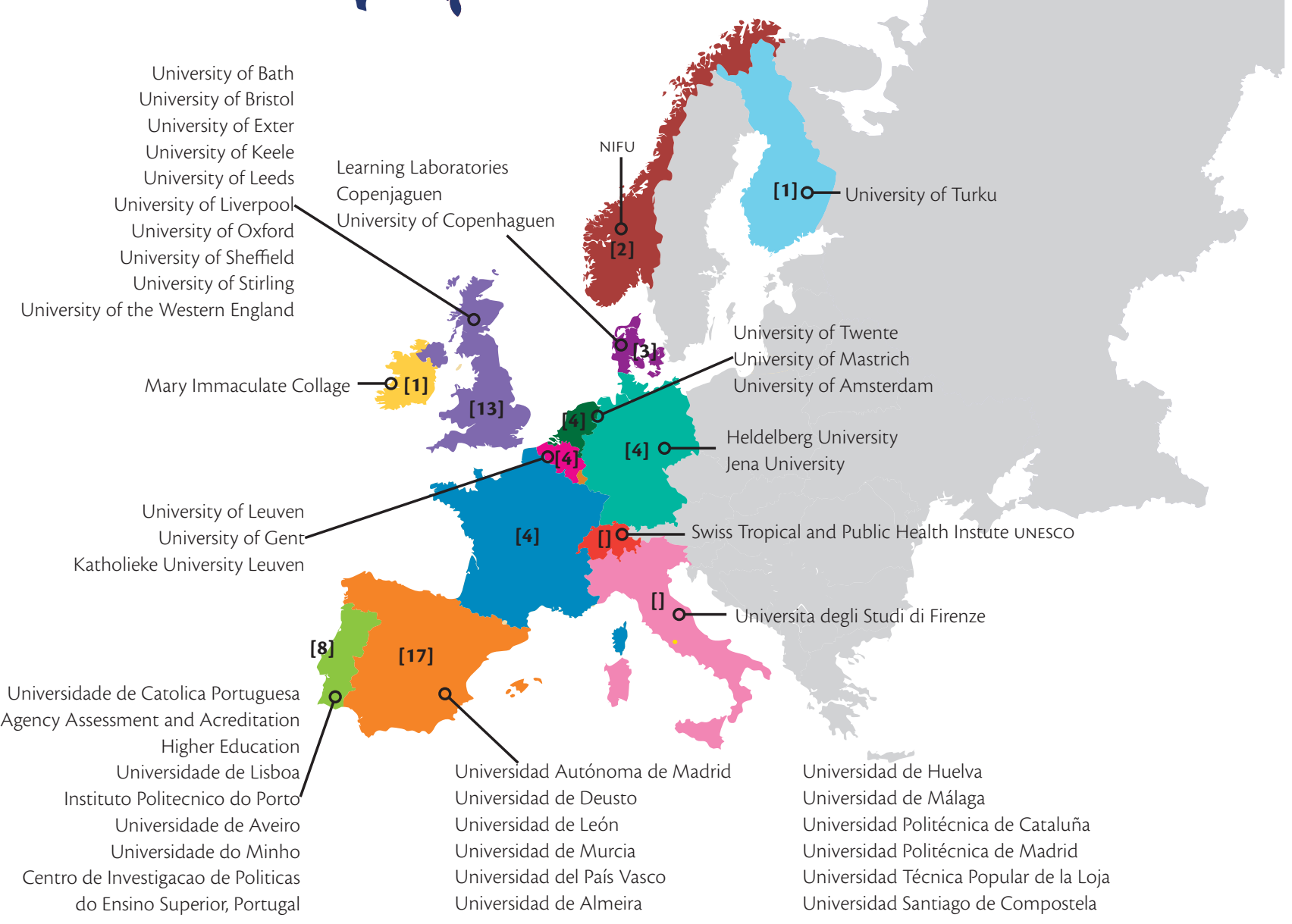

Fuente: elaboración propia 


\section{Comunidades académicas emergentes en torno a la acreditación de la calidad}

El propósito es mostrar las redes de colaboración que han emergido como resultado de los esfuerzos colectivos, sistemáticos y permanentes de las instituciones a nivel internacional en torno al tema de la acreditación. En efecto, trazar la colaboración y la coparticipación de distintos miembros académicos con base en la experiencia y las competencias permite intercambiar información valiosa para abordar el problema de la calidad con una visión disciplinar o multidisciplinar y establecer potenciales escenarios de trabajo académico.

En el siguiente apartado se presentan las formas de interrelación entre instituciones y temas, considerando las coautorías en los artículos, para comprender cómo se establecen redes de colaboración alrededor de los temas comunes a la discusión de la calidad. Un análisis general muestra que, aunque ya se logra evidenciar la aparición de comunidades, su desarrollo es embrionario y la dispersión en los temas denota bajos acuerdos entre comunidades, lo cual se evidencia en la figura 3, que agrupa por colores aquellas universidades que se interrelacionan con mayor fuerza. El tamaño de los nodos representa la cantidad de publicaciones por universidad, y la fuerza de las líneas denota la cantidad de relaciones. En este sentido, los nodos que se presentan en la figura son pequeños y las líneas de relación se muestran débiles, lo que pone en evidencia que, para el momento del análisis, aún no se mostraban comunidades o redes de trabajo sólidas en el tema. Por ende, la acreditación de la calidad como tema de interés investigativo aún es emergente.

Dentro de las primeras instituciones que muestran un interés firme en la investigación, se identificó en Europa el Centro para la Investigación de Políticas de Educación Superior (CIPES), con un número destacado de publicaciones. Por su parte, la Universidad de Toronto en Estados Unidos está liderando una de las comunidades más nutridas, seguida de la Universidad de Pennsylvania, particularmente en los temas referidos a la calidad y el mejoramiento de los currículos.

Las ocho comunidades académicas que se han venido consolidando pueden describirse así: la Comunidad 1 expuso el liderazgo de la Universidad de Toronto por contar la mayor cantidad de artículos publicados y su relación de coautoría se establece con las universidades Duke, Johns Hopkins, Oklahoma, Pittsburgh y Texas, en temas de educación superior, la internacionalización en el marco de la acreditación, la calidad de la investigación y traducción del conocimiento. La Comunidad 2, liderada por el CIPES, ha establecido lazos académicos estrechos con las universidades de Twente, Católica Portuguesa, Bath y Copenhague para el tratamiento de temas de aseguramiento de la calidad, la administración de la educación pública, los estándares y normas europeas, y los procesos de acreditación y certificación de la calidad.

La Comunidad 4 destacó el liderazgo de la Universidad RMIT en Australia en la investigación de temas relacionados con la calidad de las instituciones y los resultados de aprendizaje de los estudiantes, estableciendo vínculos fuertes con las Universidades de Wollogong, Bond y Queensland. De igual manera, la Comunidad 7, liderada por la Universidad de Penn, puso en evidencia un interés particular en estudiar las relaciones entre el currículo y la calidad.

Las Comunidades 5 y 6 se especializan en temas más específicos como la calidad de la educación médica, la calidad de la educación para el trabajo y el otorgamiento de becas en instituciones de calidad, con la participación de las universidades de Michigan, Minnesota, Alabama, Indiana, Arkansas y otras. Por otra parte, las universidades Beijing, Terbuka y Mongolia, que integran la Comunidad 8, presentan un especial interés en estudiar temas vinculados con la educación a distancia, la formación virtual y el aseguramiento de la calidad. 


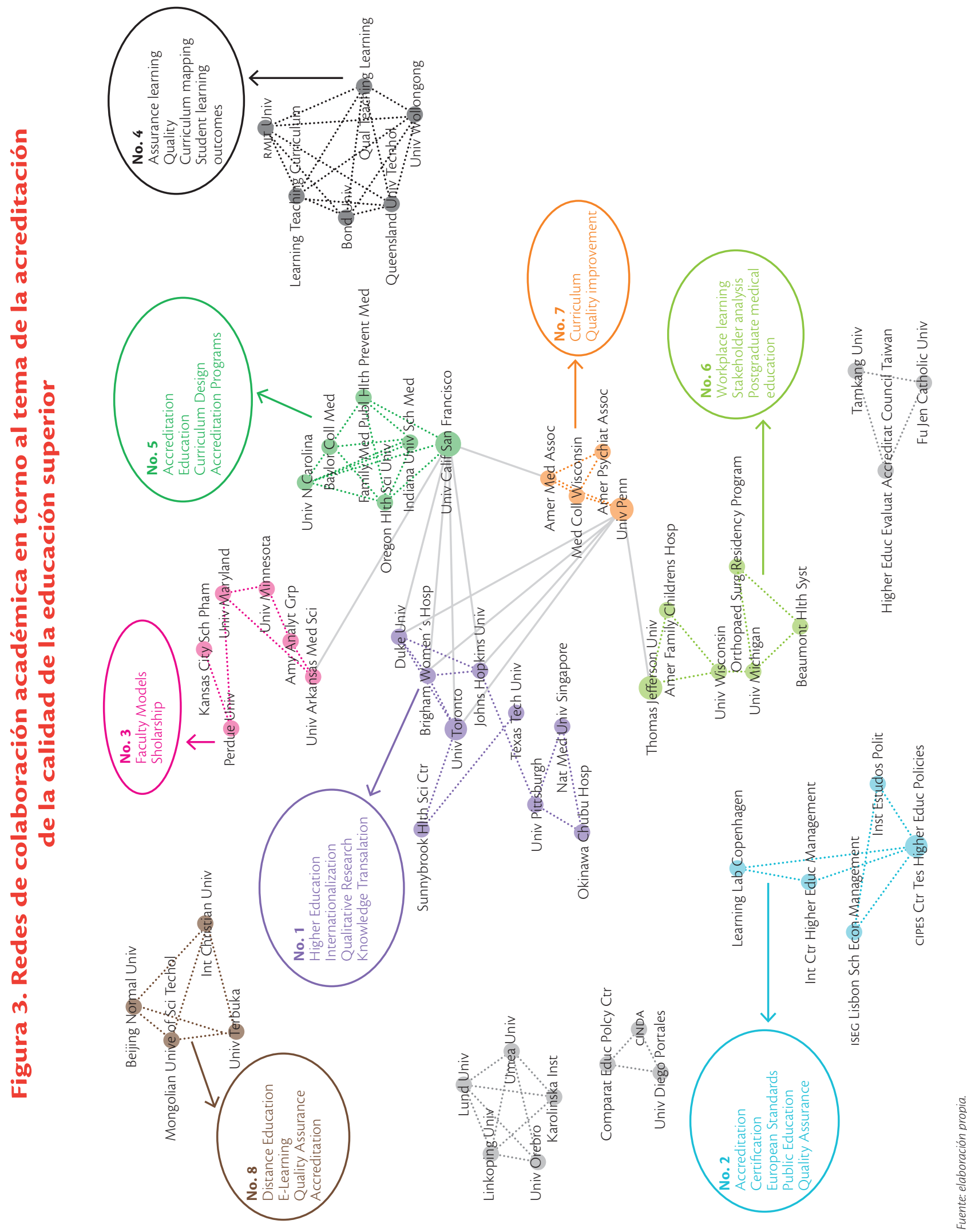




\section{La lucha de las instituciones por conseguir el prestigio de la calidad}

Primariamente, se identificó como característica común entre las instituciones descritas en las investigaciones que más allá de la preocupación por mejorar la calidad, la implementación de acreditación trajo consigo un énfasis particular en la obtención de la acreditación como un distintivo, un símbolo de prestigio y poder al interior del campo de la educación superior, en ese sentido, la acreditación ha sido entendida como una herramienta de gestión social, como un medio de legitimación frente al mercado de instituciones actual (Cret, 2010). El hecho de que distintos agentes del campo académico reconozcan la acreditación como un mecanismo legítimo para establecer diferencias entre unas instituciones y otras, supondría un propósito distinto al exhibido en los documentos de política, develaría un juego de luchas entre las instituciones para la obtención de distinciones, de reconocimiento en el campo de la educación superior que les provee beneficios diferenciales que son convertidos en capitales que consolidan su posición al interior del sistema universitario, lo que concordaría con el planteamiento de Bourdieu (2011) sobre la necesidad de admitir que "las diferencias objetivas, inscritas en propiedades materiales y en los beneficios diferenciales que proveen, son convertidas en distinciones reconocidas en y a través de representaciones que los agentes forman y realizan de ellas".

Así, conseguir la acreditación ubica a las instituciones en un lugar de reconocimiento, prestigio y legitimación, como lo demuestran Niemelä et al., (2014), preguntando a los miembros de dirección universitaria y el personal docente de una IES finlandesa si "¿vale la pena el esfuerzo de un sello de acreditación?”. Se encontró que, más allá de la búsqueda de la calidad, ellos justifican la promoción de la acreditación como un mecanismo para fortalecer la imagen pública y la visibilidad internacional de la universidad en el creciente sector universitario mundial. La acreditación es vista como una herramienta para mejorar la inclusión en redes de colaboración con otras universidades y el intercambio de estudiantes, por lo que conseguir la certificación de la acreditación, más allá de su uso para el mejoramiento de la calidad, se convierte en un boleto de entrada al mundo del prestigio internacional, equiparando a la universidad como un "par" susceptible de reconocimiento en su calidad y desempeño.

Así mismo, los procesos de acreditación de la calidad cumplen principalmente el papel de consolidar la posición institucional dentro del sector de la educación superior, y se centran en la función distintiva de las acreditaciones, caracterizadas por la obtención de un sello de calidad, como herramienta utilizada para ser reconocidos por estudiantes y recibir beneficios de los gobiernos nacionales (Cret, 2010). En este sentido, como lo mencionan Blanco y Berger (2014), la acreditación puede interpretarse como una marca, y si las marcas conectan los productos con otros productos que se parecen y a la vez que se separan de otros que son diferentes, se puede traducir que, a través del proceso de acreditación, las universidades podrían conseguir estatus y otros atributos que las unen a otras que ostentan reconocimiento y, a su vez, las distancia de aquellas que no lo poseen.

De tal manera que podría pensarse que aquellas instituciones que no logran ser acreditadas están sujetas a la inestabilidad y al fracaso (Xiaofan y Yingxia, 2014), dado que quedarán ligadas a una percepción generalizada de mediocridad, lo cual no necesariamente es cierto (Blanco-Ramírez, 2014; 2015a; 2015b; 2015c), incluso por el hecho de que "acreditarse" en algunos casos no es pertinente pues en contextos particulares, muchas universidades invertirían esfuerzos significativos para cumplir con el requisito aún sin corresponder a su propia naturaleza (Geoffroy, 2013). Prueba de ello es que la mayoría de estos estándares están sesgados considerablemente hacia los resultados de la investigación, que pueden ser apropiados para los proveedores de 
educación superior de los países desarrollados, pero tienen poco valor para las instituciones en los países en desarrollo o para Universidades de enseñanza intensiva (Pillay y Kimber, 2009). Así, la distinción de la acreditación en muchos casos puede reforzar las asimetrías existentes entre aquellas IES que cuentan con recursos adecuados y una diversificación en su cartera de indicadores, y aquellas universidades con capacidades limitadas, evocando a estas últimas para invertir grandes esfuerzos en adaptaciones foráneas que desconocen en muchos casos sus propias necesidades, y su pertinencia y responsabilidad con los contextos locales.

\section{La información y los profesores, dos capitales que definen la acreditación}

Los procesos de acreditación han propiciado que las instituciones hayan llevado a cabo grandes esfuerzos, y habría que reconocer que a muchas de ellas les ha permito incrementar sus recursos para elevar sus criterios de eficiencia, eficacia y efectividad en beneficio del mejoramiento de los procesos educativos (Suárez et al., 2007). En esa línea, Rodríguez et al. (2013) realizaron un análisis en 45 universidades chilenas, demostrando la estrecha y positiva relación entre la capacidad de gestión institucional y la calidad de la docencia e investigación, siendo éstas determinantes para la obtención de la acreditación de la calidad, demostrando que dicha obtención no sólo es producto del esfuerzo de las instituciones en elevar los indicadores, sino de su alta capacidad en la gestión de la información. En relación con esta última Fleet y Rodríguez-Ponce (2011) confirmaron que existe una tendencia que vincula significativamente la calidad de la información con la calidad de las universidades, pues una adecuada gestión de la información permite adoptar mejores decisiones para ajustar y mejorar los procesos institucionales. De hecho, aquellas instituciones que contaron con sistemas de información en los procesos de evaluación y acreditación lograron poner en evidencia una buena gestión y el enriquecimiento de los resultados para la adopción de decisiones adecuadas (Barcos, 2008), lo que apoya la tesis de que la acreditación se lee en un contexto de competición y protagonismo en el mercado de la educación superior, de tal manera que la información resulta ser un capital fundamental para la institución que sirve como eslabón entre calidad, rendición de cuentas y prestigio.

La disponibilidad de la información agrega valor en la medida que ésta se convierte en un mecanismo que permite rendir cuentas a la sociedad de forma más clara. Sin embargo, la información con la que cuentan las universidades, en la mayoría de los casos, está delimitada a indicadores de eficiencia, verbigracia, resultados de titulación, caracterización de la población estudiantil, información financiera, la matrícula, los apoyos estudiantiles, la oferta académica, etcétera, más no abundan repositorios informáticos que den cuenta de los procesos de calidad, confirmando los hallazgos de Redon-Pantoja (2009), referidos a que a pesar de que en los documentos se enuncie que la fortaleza de los procesos de acreditación radica en el significado que se le ha otorgado como mecanismo de aseguramiento de la calidad, la lógica con que opera este juicio evaluativo promueve principalmente el desarrollo de prácticas para mejorar la gestión y la visibilidad, más que las practicas académicas.

Así mismo, los diversos informes presentados por los agentes de política han puesto en evidencia que los procesos de acreditación han originado una acumulación de capitales relacionado con el factor "profesores", mostrando un alza intencional de las instituciones en las tasas de contratación y la posesión de títulos de doctorado con respecto a años anteriores a la formalización de las políticas de acreditación. Se agrega a ello, la identificación de cambios en prebendas en el tiempo de labor de los profesores para estudios de posgrado, proyectos de investigación y actividades de extensión en contextos nacionales y globales (Casas-Medina y Valdez, 2011). Esto demuestra un reconocimiento del cuerpo profesoral 
como recurso esencial, no sólo para el mejoramiento de las capacidades de los estudiantes, sino porque el sentido universitario se centra en la calidad de su práctica en la docencia, la investigación y el servicio, confirmando que la acumulación de este tipo de capital generaría una verdadera ventaja competitiva dado que las universidades que son acreditadas por más años son al parecer las que tienen una mejor dotación en cantidad y calidad de sus profesores (Pedraja-Rejas y Rodríguez-Ponce, 2014).

\section{La incorporación de la acreditación, un riesgo para la calidad docente}

Como se mencionó antes, se identifica una brecha significativa entre el planteamiento de las normas y la realidad, pues a pesar de que las reformas expresan propósitos de mejoramiento, al parecer no se evidencia un cambio significativo en las prácticas de los agentes universitarios. Al respecto, Ansah (2015) estudió en profundidad la opinión de estudiantes, administradores universitarios y profesores, en relación con los efectos de la acreditación en el mejoramiento de sus prácticas, encontrando que si bien es cierto que estos agentes reconocen que estos procesos propician una adecuada planificación, se requiere aún el desarrollo de una cultura de la calidad, que reconozca que éste es un ejercicio para mejorar y no para "cazar brujas", de lo contrario puede convertirse en un proceso sin sentido y desmotivante, que se ve reflejado en los discursos crítico-políticos que se originan en el debate mediático sobre aseguramiento de calidad en la educación superior, donde salen a relucir aspectos de inconformidad de los distintos agentes académicos, frente a diferentes temáticas que interfieren en la educación de calidad, como por ejemplo, la privatización de la educación superior y las múltiples falencias de este sector, que involucran incluso situaciones delictivas de corrupción durante el proceso de control de calidad y certificación de aseguramiento (Cabalin, 2015).

$\mathrm{Si}$ bien es cierto que los estudiantes y los administradores de universidades son agentes fundamentales, serían los profesores las fichas clave con los cuales se requiere una permanente comunicación y colaboración a lo largo del proceso (Wood, 2006). No obstante, aunque los profesores, serían el primer agente de la calidad, se dice frecuentemente que a pesar del cambio notorio en la cualificación de los profesores no se ha examinado con suficiencia sus efectos dentro de la mejora del aprendizaje de los estudiantes, empero de que los mismos modelos afirmarían que la calidad de los estudiantes es un factor determinante para explicar la calidad de las universidades. Dicho descuido se debe quizás al gran énfasis que hacen los modelos de acreditación en el factor de investigación, debido a la incipiente acumulación de este tipo de capital en el grueso de las universidades, lo que generó una tendencia creciente de las instituciones en encaminar la labor del profesor al logro de resultados en esta materia, poniendo en riesgo la atención y el esmero que mostraban por mejorar cada día más la calidad de la docencia.

Al respecto Nilsson y Walhén (2000) y Sultana et al., (2009), examinaron las políticas institucionales para mejorar el desarrollo profesional de los profesores, encontrando que los profesores piensan que llevar a cabo una buena enseñanza no los beneficia, pues no existen estímulos; los buenos maestros no son animados, pues se prefiere la investigación frente a la docencia, dejando como consecuencia una baja atención a la cualificación del quehacer pedagógico. Por ello, Larrán et al. (2013) sugieren que las instituciones deben liberar y comprometer el tiempo remunerado de los profesores, dedicado a pensar y desarrollar proyectos propios, para cualificar su práctica, lo cual constituye el mejor alimento para la calidad de su docencia. Así mismo, Borroto y Salas (1999) propondrían la inclusión de indicadores que den cuenta del nivel de competencia docente de los profesores, la calidad de la planificación en la enseñanza, el control del trabajo pedagógico, la evaluación del desempeño profesoral, entre otros. 
De otro lado, los profesores opinarían que, el hecho de que el concepto de calidad sea reconstruido para convertirse en un indicador cuantificable, que privilegia determinados tipos de conocimiento, instituciones, programas y modelos educativos, ha dejado como consecuencia una percepción negativa que se aleja de la excepcionalidad para convertirse en un requisito técnico que conlleva el mantenimiento de los estándares mínimos exigidos (Engebretsen et al., 2012). En otras palabras, como lo mencionaría Salas (2013), operacionalizar el proceso deja como consecuencia que la calidad educativa sea reducida al simple cumplimiento de indicadores y metas.

\section{La acreditación: ¿un mecanismo innecesario para el mejoramiento de la calidad?}

A partir de la pregunta sobre qué beneficios han otorgado los sistemas de acreditación a la calidad de la educación, Peña-Domínguez et al. (2005) encuentran que la acreditación ha permitido a los directivos, claustros y colectivos de estudiantes contar con un patrón de calidad con que compararse, lo que ha incrementado su motivación por alcanzar estándares de calidad que les permitan llegar a la excelencia. La cualidad más importante del proceso es la apuesta por la autoevaluación, y el hecho de que sean los propios actores quienes elaboran un plan de mejora con objetivos, acciones, responsables y fechas de cumplimiento.

Los procesos de autoevaluación, realizados de manera rigurosa, han logrado una mayor sistematización de la labor educativa y han esclarecido el quehacer propio de las universidades, lo cual es importante para el avance en los desarrollos de la ciencia y la tecnología. Estos esfuerzos han incluido revisiones y ajustes en los reglamentos internos, y procedimientos más acordes a las actividades que realizan, con lo que se genera una serie de buenas prácticas o acciones en las cuales se sustentan avances institucionales, como la modificación de los indicadores internos de calidad, resaltando así la idea de que las principales transformaciones ocurren en los ámbitos de la gestión universitaria (Lemaitre et al., 2012). También se han evidenciado beneficios tales como el aumento de los salarios de los profesores, incrementos en la productividad de la investigación y una mayor importancia percibida de las contribuciones intelectuales (Pedraja-Rejas et al., 2014). Así mismo, se han apoyado procesos para el fortalecimiento de la docencia, procesos para la disminución del índice de deserción y el establecimiento de estrategias tutoriales, asesorías e implementación de programas de apoyo académico, que logran abatir el índice de reprobación (Pedraja-Rejas y Rodríguez-Ponce, 2014).

Como contraparte, Díaz (2003-2004) analiza las diferencias entre las estrategias que ofrece la evaluación institucional como un mecanismo natural de la universidad y la acreditación de la calidad. Las considera dos herramientas distintas para promover la innovación y la mejora en la enseñanza en la educación superior, argumentando que los modelos basados en procesos naturales de autoevaluación institucional y autorregulación son más eficientes para promover mejoras en la enseñanza, siempre que éstos se realicen de forma contextualizada y con la participación de toda la comunidad educativa. La necesidad de discutir la calidad y renovar la misión, propósitos, objetivos y proyecto educativo ha sido natural en las mismas universidades, por lo cual los logros obtenidos quizás no sean un producto de la acreditación sino un resultado del mismo sentido universitario.

Sin embargo, debe reconocerse que lograr el éxito del proceso de acreditación depende ampliamente de un adecuado ejercicio de autoevaluación, el cual no es un lujo con miras a la acreditación sino un instrumento de gestión que debe aplicarse de manera orgánica en la universidad (Rodríguez et al., 2008). A su vez, la autoevaluación de las instituciones educativas permite abrir espacios para la discusión en temas como "currículo" y "pedagogía", y llegar a niveles de consenso sobre lo que se tiene y se debe hacer, 
generando nuevas preocupaciones por la calidad y la pertinencia de la educación (Rodríguez et al., 2008). Conocer logros y dificultades del proceso educativo para proponer nuevas alternativas de desarrollo que permitan el cumplimiento de la misión que ha declarado cada institución, requiere en todo caso que el proceso autoevaluativo no esté condicionado al logro de distinciones particulares, al contrario, debe ser un proceso libre de intereses y genuinamente voluntario, por lo que un adecuado proceso de autoevaluación implica que debe ser planeado de manera dinámica, continua y cíclica, con un seguimiento sistemático a las mejoras año tras año, el cual debe contar con información objetiva del quehacer institucional que permita detectar las fortalezas y debilidades a fin de que formen parte de los procesos de mejora institucional y de los cambios introducidos (Barrera y Aguado, 2007).

Sin duda, la autoevaluación es un proceso dinamizador de la calidad que contribuye a establecer las bases y las necesidades propias de la innovación para el mejoramiento institucional y la construcción de una cultura de calidad en todos los ámbitos de una institución educativa, pero sólo si éste no está condicionado o viciado bajo la presión de responder a modelos específicos, distintos a esto; la autoevaluación es inherente al propio quehacer universitario siempre que se lleve a cabo un trabajo colegiado, transparente y preciso en los procedimientos administrativos.

Bajo la misma perspectiva, Espinoza y González (2012) mencionan que los procesos de acreditación no son concebidos como una instancia destinada al mejoramiento continuo de la calidad; se perciben como opciones de control, y no constituyen un incentivo para que los actores asuman el desafio y el compromiso del mejoramiento permanente. Es importante alcanzar el mejoramiento de los criterios y de los estándares que en verdad visibilicen los avances y el impacto de las políticas, estrategias y acciones establecidas. La acreditación, más que un beneficio, puede representar un problema; dado lo intervencionista del modelo, se corre el riesgo de que las universidades se focalicen en un largo, absorbente y ambicioso trabajo conducente a la acreditación, relegando a su vez los objetivos esenciales de la educación y que terminen sirviendo a intereses sobre un mayor control del mercado educativo, con efectos que favorecen a unos pocos.

\section{Desafíos de transformación a las políticas de acreditación en Latinoamérica}

Más de allá de la conveniencia o no de los sistemas de acreditación, lo cierto es que ya forman parte de la agenda actual de las IES, razón por la cual muchos estudios concluyen enunciando distintas posibilidades para su mejoramiento. Particularmente, en países de Latinoamérica, se menciona que los sistemas aún no se han consolidado pues existe una baja apropiación, falta de claridad, sesgos económicos y poca flexibilidad de los modelos planteados (Torres-Rojas, 2012). Así mismo, no se ha logrado establecer una cultura permanente de autoevaluación al interior de las instituciones, o la construcción de indicadores que den cuenta de su realidad frente a las necesidades del contexto. Falta desarrollar una mayor preocupación por parte de los actores para mejorar los niveles de aprendizajes de los estudiantes, y las oportunidades de extender los procesos de acreditación a instituciones de diferentes estratos aún no son equitativas (De la Garza-Aguilar, 2013), en tanto existe un número considerable de instituciones y programas que aún no se han acreditados, en particular aquellos que atienden sectores de menores recursos (Espinoza y González, 2012).

Nilsson y Walhén (2000) concluyen que el sistema de acreditación requiere reformas sustanciales, particularmente en deficiencias relacionadas con la baja participación de los actores centrales del proceso (estudiantes y profesores), y modificar la atención centrada en los resultados de investigación, lo que hace que se preste menor atención al grado de involucramiento, desempeño y compromiso de los 
actores universitarios, las redes académicas y las diversas formas de aprendizaje.

Con respecto a la cobertura y eficiencia del sistema, Díaz (2003-2004) afirma que un verdadero ejercicio para acreditar los estudios universitarios supondría incorporar de manera convergente tres unidades de análisis: las instituciones, los programas de formación establecidos (planes de estudio) y el profesorado universitario. La acreditación segmentada no constituye una estrategia adecuada para promover la reflexión colectiva sobre la actividad académica, requisito imprescindible para que se puedan generar procesos de mejoramiento. Por su parte, Gómez y Celis (2009) aluden que la verificación de la calidad por parte de pares externos es de baja eficacia y de alto costo, en relación con el volumen total de programas existentes en la educación superior, por lo que se requiere con urgencia no sólo redefinir el rol y el perfil del par, sino replantear la idea de programa curricular como la unidad básica de acreditación.

La principal causa de estas ausencias se sustenta en el hecho de que no exista una diversidad de propuestas al momento de implementar una política de esta naturaleza, lo que produce no sólo un concepto de la calidad meramente técnico, sino además pone de manifiesto una concepción reduccionista que acota su uso como mecanismo de control. El análisis de la configuración de políticas de calidad en algunos países ha demostrado la ausencia de modelos de acreditación alternativos al modelo técnico planteado inicialmente; no se evidencia una posibilidad de selección entre múltiples alternativas, sino una discusión que gira siempre en torno a un mismo curso de acción técnica (Geoffroy, 2013). En casos como el de Argentina, distintos proyectos de ley tendientes a pensar en esquemas alternativos y modificar el marco normativo de la acreditación, constataron que ninguna de las diversas fuerzas políticas que tensionaban el actual modelo de evaluación de la calidad contaba con una institución que pudiera cumplir con dichas funciones (Solanas, 2011).
Esto habla mucho de la pasividad de la sociedad civil y de la falta de centros de investigación independientes que generen propuestas alternativas a las que emergen del gobierno central. De lo anterior también se concluye que la falta de discusión de alternativas, al momento de generar políticas, puede estar dando cuenta de una institucionalidad que no genera incentivos adecuados para el fortalecimiento de la democracia (Geoffroy, 2013). En este sentido, Lucasa (2013) hace un llamado para que las universidades jueguen un papel protagónico, realizando propuestas de elaboración de nuevas políticas. Sugiere igualmente que se desarrollen nuevas iniciativas para regular la educación superior, debido a que los procesos de regulación de la calidad han tenido lugar dentro de un paradigma industrial y ocupacional, y agrega la necesidad de regular la formación y actualización profesional más allá de las competencias ocupacionales. Así mismo, sería necesario que las universidades públicas, que aparecen como las grandes ausentes en este tipo de debates, tomen un liderazgo más activo en el debate de estos proyectos y puedan contribuir críticamente con esquemas alternativos (Solanas, 2011).

En consecuencia, Dill y Beerkens (2013) plantean que el desafio al que se enfrentan las naciones latinoamericanas es establecer principios marco para el diseño de políticas públicas más efectivas que apoyen a las universidades autónomas para mejorar los procesos colegiados encaminados a la calidad. La idea, de acuerdo con Escobar de Morel (2003), es proponer un marco de políticas que equilibre efectivamente las fuerzas del Estado, el mercado y la profesión académica para asegurar los estándares académicos en las universidades, así como procurar promover el análisis de problemas y el planteamiento de soluciones dentro de un contexto social, cultural y político.

\section{Discusión y conclusiones}

La exploración de este estado del arte permitió identificar a nivel internacional la configuración de al 
menos ocho comunidades académicas emergentes de investigadores e instituciones que indagan conjuntamente temas relacionados con el mejoramiento de la calidad, configurando con ello un campo de estudio que deja notar el interés compartido de un amplio grupo de académicos por conocer las implicancias de este tipo de políticas en el desarrollo de las distintas funciones universitarias.

Así mismo, se evidenció que al igual que en otros campos de la ciencia, Norteamérica muestra el liderazgo más pronunciado en la pesquisa de la calidad en la educación superior, siendo Estados Unidos el país que cuenta con el mayor número de redes establecidas para investigar sobre el tema, constatando que hay una estrecha relación entre los temas de interés por investigar en esta región con los intereses propios de desarrollo económico y político de este país. Por ejemplo, el interés por investigar el fortalecimiento de la capacidad investigativa de las instituciones, el desarrollo de la internacionalización, la calidad de la educación médica, la calidad de la educación para el trabajo, y su congruencia con estos temas vitales que se enuncian como metas en las políticas de desarrollo de esta región. De manera similar, en Europa, los académicos investigan especialmente la correspondencia entre los procesos de acreditación y los estándares de calidad establecidos para las universidades europeas, temas que se encontraron totalmente congruentes con el desarrollo notorio de esta región en la definición de un Sistema de Educación Superior Común, concretado en la Declaración de la Sorbona realizada en París el 25 de mayo de 1998, a través de la denominada "Europa del conocimiento" para la armonización y homologación de los distintos escenarios educativos, en donde la acreditación se convirtió en un mecanismo garante para la realización de dichas homologaciones, dado su carácter certificador de la calidad de los programas.

En el caso de Latinoamérica, se identificó que Chile - país que ha realizado más investigaciones en esta región - se interesa en especial en investigar temas relacionados con los componentes formativos de los programas, la calidad del cuerpo académico, y la retención de estudiantes, lo que dejaría ver una adhesión con indicadores establecidos por agencias $\mathrm{u}$ organismos patrocinadores de países en vía desarrollo, tales como el Banco Mundial, el BID o la UNESCO, cuya pertenencia a estos grupos y la existencia de lazos que los unen, permite acceder a beneficios.

De otra parte, se reconoció que el interés colectivo de participar en los procesos de acreditación, se asociaría a lo que denominaría Bourdieu el concepto de "illusio", entendido en este caso como la creencia colectiva que comparten las instituciones de educación superior sobre que vale la pena jugar el juego de la acreditación, no sólo porque así lo exige el campo sino porque se vuelve imprescindible para su permanencia en él, y se refiere al hecho de estar atrapado, de estar involucrado en el juego y por el juego, por lo que estar interesado en un juego de la acreditación implica que lo que allí ocurre tiene sentido, que sus apuestas son importantes y dignas de ser perseguidas (Bourdieu y Wacquant, 2005).

Así, la posesión de la acreditación constituye un capital simbólico de las instituciones que la obtienen porque esto las incluye en un círculo de relaciones estables que representa ventajas competitivas a partir de propiedades impalpables que se presentan como inherentes a su naturaleza, es decir, representa su talento, prestigio, reputación o fama. (Bourdieu, 2011). Este reconocimiento de la acreditación tiene una doble dimensión en el campo de la educación superior, al ser considerada como condición y, al mismo tiempo, una característica de funcionamiento de dicho campo, se trata de la respetabilidad con que cuenta una institución y que es visible para un grupo prestigioso que reconoce y aprecia la posesión de dicho capital, por tanto, se ha convertido en un bien digno de ser disputado y demostrado en el campo de la educación superior. 
De tal manera que la obtención de la acreditación provee beneficios diferenciales que, potencializados a su máxima expresión, se traducen como certificado de garantía para el Estado en cuanto a una "adecuada" utilización de los recursos, lo cual ampliaría la brecha tradicional entre universidades reconocidas y no reconocidas, favoreciendo la acumulación de capitales en aquellas que ya históricamente los poseen y dejando relegadas a aquellas que necesitan ser fortalecidas en su estructura y función.

Una interpretación adicional tiene que ver con la confirmación de que los diferentes sistemas de acreditación de la calidad implementados en distintas partes del hemisferio coinciden en que las propiedades materiales y abstractas inscritas en los procesos de acreditación se circunscriben a la disposición y cualificación de recursos, creando así una directa y estrecha relación entre la calidad educativa y la posesión de capitales de tipo social representados en los títulos de los profesores, y en la calidad de la información que posee la institución para dar cuenta de su actuar a la sociedad, dejando de lado otros determinantes de calidad relacionados con la valoración de la misma práctica educativa, así como los procesos y resultados de los modelos de enseñanza y aprendizaje que, si bien resultan complejos en su valoración, serían parte vital en la definición de la calidad educativa. 


\section{Referencias}

Ansah, Francis (2015), "A strategic quality assurance framework in an African higher education context", Quality in Higher Education, vol. 21, núm.2, pp. 132-150.

Barcos, Santiago José (2008), "Reflexiones acerca de los sistemas de información universitarios ante los desafios y cambios generados por los procesos de evaluación y acreditación”, Avaliação: Revista da Avaliação da Educação Superior, vol. 13, núm. 1, pp. 209-244.

Barrera, María Elena, Gloria Ofelia Aguado (2007), "La evaluación integral de programas y procesos: un camino hacia la calidad", EDUCERE, vol. 11, núm. 37, pp. 209-215.

Blanco-Ramirez, Gerardo (2015a), "US accreditation in Mexico: quality in higher education as symbol, performance and translation", Discourse: Studies in the Cultural Politics of Education, EDUCERE, vol. 36 núm. 3, pp. 329-342.

Blanco-Ramirez, Gerardo (2015b), "Translating quality in higher education: US approaches to accreditation of institutions from around the world", Assessment $\mathcal{E}^{\circ}$ Evaluation in Higher Education, vol. 40, núm.7, pp. 943957.

Blanco-Ramirez, Gerardo (2015c), "International accreditation as global position taking: an empirical exploration of US accreditation in México", High Education, vol. 69, pp. 361-374.

Blanco-Ramirez, Gerardo (2014), "A world of brands: higher education and the emergence of multinational quality franchises", Quality in Higher Education, vol. 20, núm. 2, pp. 216-232.

Blanco-Ramirez, Gerardo y Joseph Berger (2014), "Rankings, accreditation, and the international quest for quality: Organizing an approach to value in higher education", Quality Assurance in Education, vol. 22, núm. 1, pp. 88-104.

Borroto, Radames y Ramón Salas (1999), "El reto por la calidad y la pertinencia: la evaluación desde una visión cubana", Educación Médica Superior, vol. 13 núm. 1, pp. 80-91.
Bourdieu, Pierre (2011), Las estrategias de la reproducción social, Buenos Aires, Siglo XXI Editores.

Bourdieu, Pierre (1990), "Algunas propiedades de los campos", en Sociologíay Cultura, México, Editorial Grijalbo.

Bourdieu, Pierre (1989), "Espacio social y génesis de las clases", Estudios sobre las culturas contemporáneas, vol. 2, núm. 7, pp. 27.

Bourdieu, Pierre (1988), "Espacio social y poder simbólico", Revista de Occidente, vol. 81, pp. 97-119.

Bourdieu, Pierre y Loïc Wacquant (2005), Una invitación a la sociología reflexiva, Buenos Aires, Siglo XXI editores.

Brock, Colin (2006), "Orígenes históricos y sociales de la regulación y la acreditación de la educación superior para la garantía de la calidad", en Joaquim Tres (ed.), La educación Superior en el Mundo 2007. Acreditación para la garantía de calidad: ¿Qué está en juego.?, Madrid, Global University Network for Innovation/Mundi-Prensa, pp. 24-36

Cabalin, Cristian (2015), "Mediatizing higher education policies: discourses about quality education in the media", Critical Studies in Education, vol. 56, núm. 2, pp. 224-240.

Casas-Medina, Emma Vanesa y Erika Olivas-Valdez (2011), "El proceso de acreditación en programas de Educación Superior: un estudio de caso", Omnia, vol. 17, núm. 2, pp. 53-70.

Cret, Benoit (2010), "Accreditations as local management tools", Springer Science Business Media, vol. 61, pp. 415-429.

De la Garza-Aguilar, Javier (2013), "La evaluación de programas educativos del nivel superior en México. Avances y perspectivas", Perfiles educativos, vol. 35, pp. 33-45.

Díaz, Mario de Miguel (2003-2004), "Evaluación institucional versus acreditación, en la enseñanza superior. Implicaciones para la mejora", Contextos educativos, vol. 6, núm. 7, pp. 13-20.

Dill, D. y M. Beerkens, M. (2013), "Designing the framework conditions for assuring academic standards: lessons learned about professional, market, and 
government regulation of academic quality", High Educ, vol. 65, pp. 341-357.

Engebretsen, Eivind, Kristin Heggen y Heidi Eilertsen (2012), "Accreditation and power: a discourse analysis of a new regime of governance in higher education", Scandinavian Fournal of Educational Research, vol. 56, núm. 4, pp. 401-417.

Escobar de Morel, Margarita (2003), "Necesidad de fortalecimiento de la extensión universitaria como componente del proyecto académico, con miras a la evaluación y acreditación”, Transinformação, vol. 15, núm. 2, pp. 135-148.

Espinoza, Oscar y Luis Eduardo González (2012), "Estado actual del sistema de aseguramiento de la calidad y el régimen de acreditación en la educación superior en Chile", Revista de la Educación Superior, vol. XLI, núm. 162, pp. 87-109.

Fleet, Nicolás y Emilio, Rodríguez-Ponce (2011), "Gestión de la información y calidad de las instituciones universitarias: un estudio empírico en universidades de Chile”, Interciencia, vol. 36, núm. 8, pp. 570-577.

Geoffroy Pitta, Esteban (2013), "Estudio del sistema de aseguramiento de la calidad de la educación superior. Un análisis politológico de formulación de política pública", Revista Enfoques: Ciencia Política y Administración Pública, vol. XI, núm. 19, pp. 139-166.

Giddens, Anton, (1984), La constitución de la sociedad, Barcelona, Alianza Editorial.

Giner, Salvador (2001), Teoría social clásica, Barcelona, Ariel.

Ginkel, Hans J. y Marco Antonio Dias (2007), "Retos institucionales y políticos de la acreditación en el ámbito internacional", en Joaquim Tres (ed.), La educación superior en el mundo 2007. Acreditación para la garantía de calidad: ¿qué está en juego?, Madrid, Global University Network for Innovation/Mundi-Prensa, pp. 58-72.

Gómez, Victor y Jorge Celis (2009), "Sistema de aseguramiento de la calidad de la educación superior: consideraciones sobre la acreditación en Colombia,
Revista Colombiana de Sociología, vol. 32, pp. 87-110.

Gutiérrez, Alicia (2012), Las prácticas sociales. Una introducción a Pierre Bourdieu, Villa María, Argentina, Editorial Eduvim. Larrán, Jorge, M., Bernabe Escobary Emma García(2013), "El sistema de acreditación nacional: la opinión de los profesores universitarios de contabilidad", Revista Española de Documentación Científica, vol. 36, núm. 3, pp. e015.

Lemaitre, María José, Mario Maturana, Elisa Zenteno y Andrea Alvarado (2012), "Cambios en la gestión institucional en universidades, a partir de la implementación del sistema nacional de aseguramiento de la calidad: la experiencia chilena", Calidad en la Educación, vol. 36, pp. 21-52.

Lucasa, Norman (2013), "One step forward, two steps back? The professionalisation of further education teachers in England", Research in Post-Compulsory Education, vol. 18, núm. 4, pp. 389-401.

Niemelä, Hanna, Taija Okkola, Aannikka Nurkka, Mikko Kuisma y Ritva Tuunila (2014), "Is an accreditation seal worth the effort?: observations of programme accreditations in Lappeenranta University of Technology, Finland", Quality Assurance in Education, vol. 22, núm. 3, pp. 226-239.

Nilsson, Karl y Staffan Wahlén (2000), "Institutional response to the Swedish model of quality assurance", Quality in Higher Education, vol. 6, núm. 1, pp. 7-18.

Pedraja-Rejas, Liliana y Emilio Rodríguez-Ponce (2014), "Análisis del impacto de los recursos organizativos sobre la calidad de las universidades: un estudio cuantitativo del caso chileno”, Interciencia, vol. 39, núm. 10, pp. 697-703.

Peña-Domínguez, Misdalia, Juan Fernando Peña-Santín, Leticia Ferrando-Alonso, y Niurka Reyes-Chávez (2005), "La acreditación de carreras universitarias. Experiencia cubana", Innovación Educativa, vol. 5, núm. 29, pp 21-35.

Pillay, Hitendra y Megan Kimber (2009), "Quality assurance in higher education: for whom and of what?", International fournal of Management in Education, vol. 3, núm. 4, pp. 270-281. 
Redon-Pantoja, Silvia (2009). "Auto evaluación institucional y acreditación como aseguramiento de la calidad de la educación: implicancias teóricas y prácticas", Estudios Pedagógicos, vol. XXV, núm. 2, pp. 269-284.

Rodman, Karmen, Rodman Biloslavo, y Silva Bratož (2013), "Institutional quality of a higher education institution from the perspective of employers", Minerva, vol. 51, pp. 71-92.

Rodríguez-Ponce, Emilio, Liliana Pedraja-Rejas, Carmen Araneda y Juan Rodríguez-Ponce (2013), "Relaciones entre los determinantes de la calidad de las universidades: un estudio exploratorio desde Chile", Revista de Ciencias Sociales, vol. XIX, núm. 3, pp. 446-456.

Rodríguez, Ulises, Claudia Zúñiga y Elizabeth Arnáez (2008), "Factores que contribuyen con el éxito en los de autoevaluación con miras a la acreditación. Experiencia del Instituto Tecnológico de Costa Rica", Actualidades Investigativas en Educación, vol. 8, núm. 1, pp. 1-99.

Salas, Iván (2013), "La acreditación de la calidad educativa y la percepción de su impacto en la gestión académica: el caso de una institución del sector no universitario en México", Calidad en la Educación, vol. 38, pp. 305-333.

Solanas, Facundo (2011), "La ley de educación superior en Argentina y la institucionalización de la acreditación universitaria: de los cuestionamientos a las dificultades del cambio", Universidades, vol. LXI, núm. 51, pp. 29-42.

Suárez-Rosas, Luis, Irene Barrios-Osuna y María
González-Espíndola (2007), "Sistema de evaluación y acreditación de carreras universitarias: implantación en el Instituto Superior de Ciencias Médicas de La Habana", Revista Habanera de Ciencias Médicas, vol. 6, núm. 3, pp.1-11.

Sultana, Naveed, Yousuf Imran, Muhammad Naseer y Sajid Rehman (2009), "The higher the quality of teaching", Contemporary Issues in Education Research, vol. 2, núm. 3, pp. 59-64.

Torres-Rojas, Emilio (2012), "Acreditación institucional y la mirada de los actores. Un estudio cualitativo en universidades privadas de Santiago", Estudios Pedagógicos, vol. 38, núm. 2, pp. 221-242.

Uvalić-Trumbić, Stamenka (2006), "Política internacional de garantía de la calidad y acreditación: de los instrumentos legales a las comunidades de práctica”, en Joaquim Tres (ed.), La educación superior en el Mundo 2007. Acreditación para la garantía de calidad: ¿qué está en juego?, Madrid, Global University Network for Innovation/ Mundi-Prensa, pp. 37-57.

Wood, Ann L. (2006), "Demystifying accreditation: action plans for a national or regional accreditation", Innovative Higher Education, vol. 31, núm. 1, pp. 43-62.

Xiaofan, Li y Cao Yingxia (2014), "Quality and quality assurance in Chinese private higher education", Quality Assurance in Education, vol. 22, núm.1, pp. 65-87.

Cómo citar este artículo:

Guzmán-Puentes, Sandra y René Guevara-Ramírez (2022), “Configuración de la acreditación de la calidad como campo de estudio. Una revisión sistemática de la investigación internacional (1998-2016)", Revista Iberoamericana de Educación Superior (RIES), vol. XIII, núm. 36, pp. 160-180, DoI: https://doi.org/10.22201/iisue.20072872e.2022.36.1189 [Consulta: fecha de última consulta]. 